\title{
BMJ Open Prevalence of lower urinary tract symptoms suggestive of benign prostatic hyperplasia (LUTS/BPH) in China: results from the China Health and Retirement Longitudinal Study
}

\author{
Weiyu Zhang, ${ }^{1}$ Xiaopeng Zhang, ${ }^{1}$ Haibin $\mathrm{Li},{ }^{2}$ Feng Wu, ${ }^{3}$ Huanrui Wang, ${ }^{1}$ \\ Meishan Zhao, ${ }^{4} \mathrm{Hao} \mathrm{Hu},{ }^{1} \mathrm{Kexin} \mathrm{Xu}^{1}$
}

To cite: Zhang W, Zhang $X$, $\mathrm{Li} \mathrm{H}$, et al. Prevalence of lower urinary tract symptoms suggestive of benign prostatic hyperplasia (LUTS/BPH) in China: results from the China Health and Retirement Longitudinal Study. BMJ Open 2019;9:e022792. doi:10.1136/ bmjopen-2018-022792

- Prepublication history for this paper is available online. To view these files, please visit the journal online (-2018022792">http://dx.doi.org/10. 1136/bmjopen-2018-022792)

WZ and $X Z$ contributed equally.

Received 10 March 2018

Revised 7 March 2019

Accepted 27 March 2019

Check for updates

(c) Author(s) (or their employer(s)) 2019. Re-use permitted under CC BY-NC. No commercial re-use. See rights and permissions. Published by BMJ.

For numbered affiliations see end of article.

Correspondence to Professor $\mathrm{HaO} \mathrm{Hu}$; huhao@bjmu.edu.cn and Professor Kexin Xu; cavinx@yeah.net

\section{ABSTRACT}

Objective Rapid population ageing in China is increasing the prevalence of lower urinary tract symptoms suggestive of benign prostatic hyperplasia (LUTS/BPH) among older people. The associated economic burden is increasing as well. Relevant data from China are currently insufficient.

Design Secondary analysis of a cohort sample.

Setting A nationally representative, cross-sectional survey - the China Health and Retirement Longitudinal Study (CHARLS) — was conducted in 2011 in mainland China.

Participants The study included individuals in the community selected from CHARLS by multistage probability sampling. A total of 5888 participants aged 50 years and above were included.

Outcome measures Self-reported morbid state was derived from a structured questionnaire. The weighted prevalence of LUTS/BPH was estimated and stratified by age group, marital status, education level, economic level, residential area and geographical region. Multivariable weighted logistic regression was used to examine the association of socioeconomic status with the odds of $\mathrm{BPH}$. Results The weighted overall prevalence of LUTS/BPH was $10.66 \%$ (95\% Cl 9.36 to 12.12). Among individuals aged over 70 years, the prevalence was $14.67 \%(95 \% \mathrm{Cl}$ 11.80 to 18.09$)$ and it increased with ageing $(p<0.05)$. The prevalence of LUTS/BPH among subjects residing in urban areas was higher $(13.55 \%, 95 \% \mathrm{Cl} 10.95$ to 16.64$)$ than those living in rural areas $(8.38 \%, 95 \% \mathrm{Cl} 6.90$ to 10.15). The prevalence of LUTS/BPH was lowest in the South-Central and South-West regions and highest in the North-West region.

Conclusions We found an increasing trend of prevalence of LUTS/BPH with ageing. It varied according to marital status, socioeconomic status and geographical region.

Benign prostatic hyperplasia $(\mathrm{BPH})$ is a common disease in men representing a substantial disease burden. BPH is characterised by a proliferation of both the stromal and epithelial cells of the prostate in the transitional zone surrounding the urethra.
Strengths and limitations of this study

- Our data are based on the China Health and Retirement Longitudinal Study (CHARLS), a strict national population survey.

- The prevalence of lower urinary tract symptoms suggestive of benign prostatic hyperplasia (LUTS/BPH) in China is difficult to estimate because of the large population and of the diagnostic criteria.

- CHARLS did not collect weight and height, so we could not analyse the relationship of body mass index and obesity with the prevalence of LUTS/BPH.

Approximately $50 \%$ of men $>50$ years of age have pathological evidence of $\mathrm{BPH}$, increasing to $>80 \%$ as men reach their eighth decade of life and older. ${ }^{2}$ When men reached 80 years, this number increases to $83 \% .^{3}$ As the world population aged, the incidence and prevalence of $\mathrm{BPH}$ have rapidly increased. ${ }^{4}$ Lower urinary tract symptoms (LUTS) have been specified by the standardisation subcommittee of the International Continence Society in February 2002: 'LUTS are the subjective indicators of a disease or change in conditions as perceived by the patients, carer or partners and may lead him/ her to seek help from health care professionals. Symptoms may either be volunteered or described during the patient interview. They are usually qualitative'. However, in 2006, Chapple and Roehrborn ${ }^{6}$ emphasised that the presence of LUTS does not need to be associated with prostatic pathology only. Therefore, LUTS/BPH in our study referred to symptomatic BPH. Although it is not life-threatening, LUTS/BPH is associated with serious morbidities and decreases quality of life. ${ }^{78}$ In America, LUTS/BPH affects more than $20 \%$ of American men aged 30-79 
years, or roughly 15 million men. ${ }^{9} 10$ The prevalence of BPH in China is currently determined from autopsy data and a few studies based on the general population that cannot accurately reflect the current status of the disease. ${ }^{11}$ No study has been conducted on LUTS/BPH in China. Therefore, there is a paucity of data on the prevalence of LUTS/BPH and its potential risk factors among the elderly in China.

China has a population of 1.3 billion, $25.3 \%$ aged 50 years or older and $13.26 \%$ aged more than 60 years. ${ }^{12}$ The ageing of the general population means that elderly people now account for a much greater proportion of patients with BPH. Many modifiable risk factors play roles in the pathogenesis of $\mathrm{BPH}$, including sex steroid hormones, the metabolic syndrome cardiovascular disease, obesity, diabetes, diet, physical activity and inflammation. These risk factors cause a large variation in the prevalence of LUTS/BPH in various regions of China. Epidemiological studies comparing the prevalence of LUTS/BPH according to age, socioeconomic status and geographical region by the same research method will provide reliable estimates on the understanding of potential risk factors of LUTS/BPH and help design healthcare plans.

Using data collected from the China Health and Retirement Longitudinal Study (CHARLS), a national random sample of the Chinese population, ${ }^{13}$ we estimated the prevalence of LUTS/BPH among residents aged 50 years or older in China according to age, marital status, education level, geographical region and socioeconomic status.

\section{METHODS}

CHARLS is a survey of the elderly in China based on a sample of households with members who are middleaged and elderly and their spouses. Individuals aged 50 years and above were included in this study. Data on socioeconomic and health status were collected using standardised questionnaire. ${ }^{13}$ The baseline survey was conducted in 2011-2012, covering 450 villages/urban communities in 28 provinces. Eligible individuals were selected through four-stage, stratified cluster sampling. Probabilities proportional to size sampling was used to determine sample size. Detailed descriptions are provided in a previous publication. ${ }^{14}$

\section{Definitions of LUTS/BPH}

$\mathrm{BPH}$ is a term reserved for the typical histological pattern that defines the disease. However, many men with histological BPH never seek medical care, nor do they require treatment for it. The condition proceeds differently when it is associated with LUTS. Individuals with histological $\mathrm{BPH}$ visit a doctor only when LUTS occurs. Residents who participated in CHARLS were asked whether they have ever been diagnosed with a prostate illness (excluding prostatic cancer). We defined him as a subject with LUTS/BPH if he responded positively to this question.

\section{Data collection and grouping}

Households were selected randomly and age-eligibly. All participants had a face-to-face household interview using a structured questionnaire. Information collected during the household interview included demographic factors, socioeconomic status and medical history. The results of the statistical analysis will not be disseminated to study participants in a short time.

Subjects were grouped into three strata according to age: 50-59, 60-69 and $\geq 70$ years old. Marital status was divided into married and unmarried/separated/ widowed. Education level was divided into five categories: no formal education, elementary school, middle school, high school, and college degree or above. Geographical region was divided into rural and urban. Economic level was defined according to the tertile of gross domestic product (GDP). We categorised their living localities into six regions, that is, East (seven provinces: Shandong, Jiangsu, Shanghai, Zhejiang, Fujian, Anhui and Jiangxi), North (two cities: Beijing and Tianjin; three provinces: Hebei, Shanxi and Inner Mongolia), North-East (three provinces: Liaoning, Jilin and Heilongjiang), North-West (four provinces: Shaanxi, Gansu, Qinghai and Xinjiang), South-Central (five provinces: Henan, Hubei, Hunan, Guangdong and Guangxi) and South-West (one city: Chongqing; three provinces: Sichuan, Guizhou and Yunnan). Individuals living in Hainan, Ningxia, Taiwan and Tibet were not selected in this survey.

\section{Patient and public involvement}

Patients and/or the public were not involved in the design or conduct of this study.

\section{Statistical analyses}

We used Stata to calculate the overall and age-specific prevalence of LUTS/BPH according to strata for each factor. The svy: logistic procedure in Stata V.14.2 was used to examine the association between each risk factor and the prevalence of BPH, adjusting for other potential confounders including gender, age, area, education, GDP per capita and region. Both procedures considered the complex survey design and the non-response rate for the CHARLS survey when estimating the prevalence, prevalence OR and corresponding SE.

\section{RESULTS}

A total of 5888 participants were involved in our study and the characteristics of the baseline population are given in table 1 . Five hundred and ninety-nine responded positively. The mean age was 62.78 (SD: 8.56). Most of the participants were married. About a quarter of the participants did not receive formal education and half of the rest received elementary or middle school education. The majority of the respondent participants lived in rural areas. About one-third of the respondents were classified in three levels of GDP per capita. 
Table 1 Baseline population characteristics

\begin{tabular}{|c|c|}
\hline Characteristics & Men $(n=5888)$ \\
\hline Age, mean (SD) & $62.78(8.56)$ \\
\hline \multicolumn{2}{|l|}{ Age group, n (\%) } \\
\hline $50-59$ & 2356 (40.01) \\
\hline $60-69$ & $2250(38.21)$ \\
\hline$\geq 70$ & $1282(21.77)$ \\
\hline \multicolumn{2}{|l|}{ Marital status, n (\%) } \\
\hline Married & $5111(86.80)$ \\
\hline Unmarried/Separated/Widowed & $777(13.20)$ \\
\hline \multicolumn{2}{|l|}{ Education level, $n(\%)^{*}$} \\
\hline No formal education & $1918(32.84)$ \\
\hline Elementary school & $1573(26.93)$ \\
\hline Middle school & $1426(24.42)$ \\
\hline High school & $556(9.52)$ \\
\hline College degree or above & $367(6.28)$ \\
\hline \multicolumn{2}{|l|}{ Geographical region, n (\%) } \\
\hline Rural & $3742(63.55)$ \\
\hline Urban & $2146(36.45)$ \\
\hline \multicolumn{2}{|l|}{ GDP per capita, n (\%) } \\
\hline Low & $2114(35.90)$ \\
\hline Middle & 1960 (33.29) \\
\hline High & $1814(30.81)$ \\
\hline \multicolumn{2}{|l|}{ Region, n (\%) } \\
\hline East & 2506 (42.56) \\
\hline North & $779(13.23)$ \\
\hline North-East & $404(6.86)$ \\
\hline North-West & $224(3.80)$ \\
\hline South-Central & $974(16.54)$ \\
\hline South-West & $1001(17.00)$ \\
\hline
\end{tabular}

${ }^{\star}$ Education level values of 48 cases were missed.

GDP, gross domestic product.

The weighted overall prevalence of LUTS/BPH was $10.66 \%$ (95\% CI 9.36 to 12.12). The weighted results are listed in table 2.

\section{Age}

Ageing men were more likely to be diagnosed with LUTS/BPH, and the weighted prevalence of LUTS/BPH increased with age. Compared with subjects aged $<60$ years, the adjusted ORs for the prevalence of LUTS/BPH were 1.50 (95\% CI 1.20 to 1.87 ) for those aged 60-69 years and 2.09 (95\% CI 1.58 to 2.78) for those aged $\geq 70$ years (table 3 ).

\section{Marital status}

The prevalence of LUTS/BPH was slightly higher among married individuals, at $10.80 \%$ (95\% CI 9.61 to 12.12), while the prevalence among unmarried/separated/ widowed individuals was $9.87 \%$ (95\% CI 6.56 to 14.60).
The odds of LUTS/BPH in unmarried/separated/ widowed individuals were approximately 0.91 times that of married individuals.

\section{Education and economic levels}

There was variation in the prevalence of LUTS/BPH among various education and economic levels. The prevalence was higher among individuals with more years of education and it was highest among individuals with college degrees or above $(p<0.05)$. The prevalence of LUTS/BPH was lower among individuals with a low GDP per capita.

\section{Resident areas and regions}

The prevalence of LUTS/BPH was higher among subjects residing in urban areas (rural: $8.38 \%, 95 \%$ CI 6.90 to 10.15; urban: $13.55 \%, 95 \%$ CI 10.95 to 16.640 ). The odds of LUTS/BPH in urban residents were 1.5 times that of rural residents. There was a significant difference in the prevalence of LUTS/BPH according to geographical location. The South-Central and South-West regions had the lowest prevalence of LUTS/BPH $(9.76 \%$ and $9.53 \%$, respectively), followed by the East $(10.56 \%)$, North $(12.18 \%)$ and North-East $(12.24 \%)$ regions; the prevalence was highest among respondents living in the NorthWest region $(13.75 \%)$. Compared with the East region, the ORs for LUTS/BPH for the North, North-East, NorthWest, South-Central and South-West regions were 1.12 (95\% CI 0.75 to 1.67 ), 1.03 (95\% CI 0.76 to 1.39 ), 1.66 (95\% CI 1.12 to 2.47 ), 0.90 (95\% CI 0.53 to 1.55 ) and 1.01 (95\% CI 0.66 to 1.53), respectively, after adjustment for age, marital status, rural/urban area, education and GDP per capita (table 3). The prevalence in each region can be seen in figure 1 .

\section{DISCUSSION}

CHARLS set up a high-quality, nationally representative, publicly available micro-database providing a wide range of information on the households of the elderly, as well as individual information on the elderly respondents and their spouses. Our research is based on data from CHARLS. It was known from our study that LUTS/BPH was common among Chinese men aged over 50 years. Age had a great influence on the prevalence of LUTS/ $\mathrm{BPH}$ according to ours and other studies. ${ }^{15}$

Rapid population ageing in China is increasing the prevalence of LUTS/BPH among older people and so does the economic burden associated with it. In our study, the adjusted prevalence of LUTS/BPH among men aged beyond 50 years reached $10.66 \%$ (95\% CI 9.36 to 12.12 ), which could influence the quality of their life and impose economic burden on both individuals and society. The cost of intervention and treatment of BPH comprised direct costs (drugs, procedures, imaging and office visits), indirect costs (lost earnings) and intangible costs (pain and suffering). ${ }^{16}$ It is likely that the cost of LUTS/BPH will continuously increase in the future. Therefore, a 
Table 2 Weighted prevalence

\begin{tabular}{|c|c|c|c|}
\hline Characteristics & Case, n (\%) & Prevalence $(\%)$ & $95 \% \mathrm{Cl}$ \\
\hline Total & $599(10.17)$ & 10.66 & 9.36 to 12.12 \\
\hline \multicolumn{4}{|l|}{ Age group } \\
\hline $50-59$ & $182(7.72)$ & 8.39 & 7.01 to 9.98 \\
\hline $60-69$ & $236(10.49)$ & 10.61 & 8.67 to 12.91 \\
\hline$\geq 70$ & $181(14.12)$ & 14.67 & 11.80 to 18.09 \\
\hline \multicolumn{4}{|l|}{ Marital status } \\
\hline Married & $522(10.21)$ & 10.80 & 9.61 to 12.12 \\
\hline Unmarried/Separated/Widowed & 77 (9.91) & 9.87 & 6.56 to 14.60 \\
\hline \multicolumn{4}{|l|}{ Education level ${ }^{*}$} \\
\hline No formal education & $162(8.45)$ & 8.20 & 6.54 to 10.23 \\
\hline Elementary school & $166(10.55)$ & 10.69 & 8.20 to 13.83 \\
\hline Middle school & $130(9.12)$ & 9.17 & 6.71 to 12.40 \\
\hline High school & $55(9.89)$ & 13.99 & 7.62 to 24.29 \\
\hline College degree or above & $84(22.89)$ & 21.26 & 13.55 to 31.73 \\
\hline \multicolumn{4}{|l|}{ Geographical region } \\
\hline Rural & $328(8.77)$ & 8.38 & 6.90 to 10.15 \\
\hline Urban & $271(12.63)$ & 13.55 & 10.95 to 16.64 \\
\hline \multicolumn{4}{|l|}{ GDP per capita } \\
\hline Low & $203(9.60)$ & 10.69 & 8.20 to 13.83 \\
\hline Middle & $232(11.84)$ & 11.54 & 10.09 to 13.16 \\
\hline High & $164(9.04)$ & 9.83 & 7.44 to 12.88 \\
\hline \multicolumn{4}{|l|}{ Region } \\
\hline East & $244(9.74)$ & 10.56 & 8.68 to 12.81 \\
\hline North & $98(12.58)$ & 12.18 & 8.63 to 16.92 \\
\hline North-East & $42(10.40)$ & 12.24 & 9.11 to 16.25 \\
\hline North-West & 29 (12.95) & 13.75 & 9.92 to 18.75 \\
\hline South-Central & $91(9.34)$ & 9.76 & 6.47 to 14.45 \\
\hline South-West & 95 (9.49) & 9.53 & 6.24 to 14.31 \\
\hline
\end{tabular}

${ }^{\star}$ Education level values of 48 cases were missed. GDP, gross domestic product.

prevalence study on LUTS/BPH can help guide public health policy.

As the definition of $\mathrm{BPH}$ varies, the prevalence varies. ${ }^{17}$ When responding positively to a question on the diagnosis of a benign prostate illness, an individual means that some symptoms of LUTS occurred in him, and that he sought for medical care and was diagnosed with a benign prostate illness. Benign prostate illness primarily refers to prostatitis and $\mathrm{BPH}$, which shared many overlapping symptoms. Prostatitis without BPH is a diagnosis in young men; however, inflammation in the prostate is also observed in elderly men presenting with BPH. Gandaglia et $a l^{18}$ suggested that histological prostatitis affected the progression of BPH because of the inflammatory process. Inflammation is a modifiable risk factor in $\mathrm{BPH}$ pathogenesis. ${ }^{15}$ Patients presenting with BPH may have a component of category IV, asymptomatic prostatitis. ${ }^{19}$ Thus, in our study, individuals over aged 50 who responded positively to the question on diagnosis with a benign prostate illness were counted as having LUTS/BPH.

$\mathrm{BPH}$ increases with age, as has been confirmed by numerous studies. Loeb et $a t^{20}$ enrolled 278 men from the Baltimore Longitudinal Study of Aging and reported that the median rate of volume change was 0.6 cc per year (range -9.9 to 62.1 ), corresponding to a median growth rate of $2.5 \%$ per year (range $-29.2 \%$ to $176.4 \%$ ). The study was based on autopsy. Although prostate volume is not related to LUTS severity directly, it is a risk factor. Roehrborn $e t a l^{21}$ reported that a larger prostate was associated with an increased risk of urinary retention, increased future need for surgery and clinical progression of BPH. Another study, after a follow-up of 16 years, reported significantly increased incidence and progression of LUTS in older men. ${ }^{22}$ In our study, LUTS/BPH involved 


\begin{tabular}{|c|c|c|c|}
\hline Characteristics & Crude OR $(95 \% \mathrm{Cl})$ & Adjusted OR (95\% Cl) & $P$ value \\
\hline Age group & & & $<0.001$ \\
\hline $50-59$ & 1.00 (reference) & 1.00 (reference) & \\
\hline $60-69$ & 1.29 (0.98 to 1.71$)$ & 1.50 (1.20 to 1.87$)$ & 0.001 \\
\hline$\geq 70$ & 1.87 (1.31 to 2.69 ) & 2.09 (1.58 to 2.78 ) & $<0.001$ \\
\hline \multicolumn{4}{|l|}{ Marital status } \\
\hline Married & 1.00 (reference) & 1.00 (reference) & \\
\hline Unmarried/Separated/Widowed & 0.90 (0.59 to 1.38$)$ & 0.91 (0.63 to 1.30$)$ & 0.597 \\
\hline Education level & & & 0.001 \\
\hline No formal education & 1.00 (reference) & 1.00 (reference) & \\
\hline Elementary school & 1.34 (0.95 to 1.88$)$ & 1.36 (0.97 to 1.91$)$ & 0.074 \\
\hline Middle school & $1.12(0.79$ to 1.61$)$ & 1.30 (0.93 to 1.82$)$ & 0.120 \\
\hline High school & 1.82 (0.84 to 3.92$)$ & 2.27 (1.03 to 4.99$)$ & 0.043 \\
\hline College degree or above & 3.02 (1.67 to 5.43 ) & 2.67 (1.57 to 4.54$)$ & 0.000 \\
\hline \multicolumn{4}{|l|}{ Geographical region } \\
\hline Rural & 1.00 (reference) & 1.00 (reference) & \\
\hline Urban & 1.71 (1.24 to 2.36$)$ & 1.50 (1.71 to 1.92$)$ & 0.002 \\
\hline GDP per capita & & & 0.156 \\
\hline Low & 1.00 (reference) & 1.00 (reference) & \\
\hline Middle & 1.08 (0.77 to 1.53$)$ & 1.23 (0.94 to 1.62$)$ & 0.130 \\
\hline High & 0.91 (0.58 to 1.42$)$ & 0.99 (0.69 to 1.42$)$ & 0.958 \\
\hline Region & & & 0.200 \\
\hline East & 1.00 (reference) & 1.00 (reference) & \\
\hline North & 1.17 (0.74 to 1.84$)$ & 1.12 (0.75 to 1.67$)$ & 0.578 \\
\hline North-East & $1.18(0.77$ to 1.79$)$ & 1.03 (0.76 to 1.39$)$ & 0.844 \\
\hline North-West & 1.35 (0.86 to 2.11$)$ & 1.66 (1.12 to 2.47$)$ & 0.012 \\
\hline South-Central & 0.91 (0.55 to 1.51$)$ & 0.90 (0.53 to 1.55$)$ & 0.696 \\
\hline South-West & 0.89 (0.53 to 1.50$)$ & 1.01 (0.66 to 1.53$)$ & 0.970 \\
\hline
\end{tabular}

$\mathrm{BPH}$, benign prostatic hyperplasia; LUTS, lower urinary tract symptoms.

symptoms of LUTS and histological BPH. Individuals aged 60-69 years and $\geq 70$ years had a higher prevalence of LUTS/BPH, and the adjusted ORs were 1.5 (95\% CI 1.20 to 1.87 ) and 2.09 (95\% CI 1.58 to 2.78 ), respectively, compared with subjects aged $<60$ years $(\mathrm{p}<0.05)$.

Studies on sex steroid hormones and BPH revealed that androstanediols play a role in BPH development. ${ }^{23}$ Unmarried/separated/widowed individuals accounted for $13.20 \%$ in all the respondent participants, having a lower adjusted prevalence of LUTS/BPH at $9.87 \%(95 \%$ CI 6.56 to 14.60). These people are usually thought to have no or less sexual life compared with married men. Married men more often intended to visit a doctor in case their illness would have a negative impact on their spouse and family.

In several studies, socioeconomic status played an important role in the progression of LUTS/BPH and might vary the results, particularly the prevalence and effects of LUTS/BPH. ${ }^{24}$ For example, some researchers found higher rates of $\mathrm{BPH}$ in upper income groups, but this may be due to selection bias, because of higher utilisation of medical care. ${ }^{25}{ }^{26}$ Using data from the Korean Community Health Survey performed in 2011, Jo et $a l^{27}$ found that the severity of LUTS was associated with several socioeconomic factors, including education level, income level and living environment. Fowke $e t a t^{28}$ found that college education or higher level was associated with a lower international prostate symptom score (IPSS) score. Education levels were obtained when people were young, and did not change with ageing. Nevertheless, education influenced the understanding of the disease and the decision-making progress. In our study, we analysed socioeconomic factors including GDP per capita, education levels and geographical region. Individuals with college degree or above had a high adjusted OR of LUTS/BPH at 2.67 (95\% CI 1.57 to 4.54$) \quad(\mathrm{p}<0.001)$, compared with individuals with no formal education. Individuals residing in urban areas were 1.5 times more likely to have the 


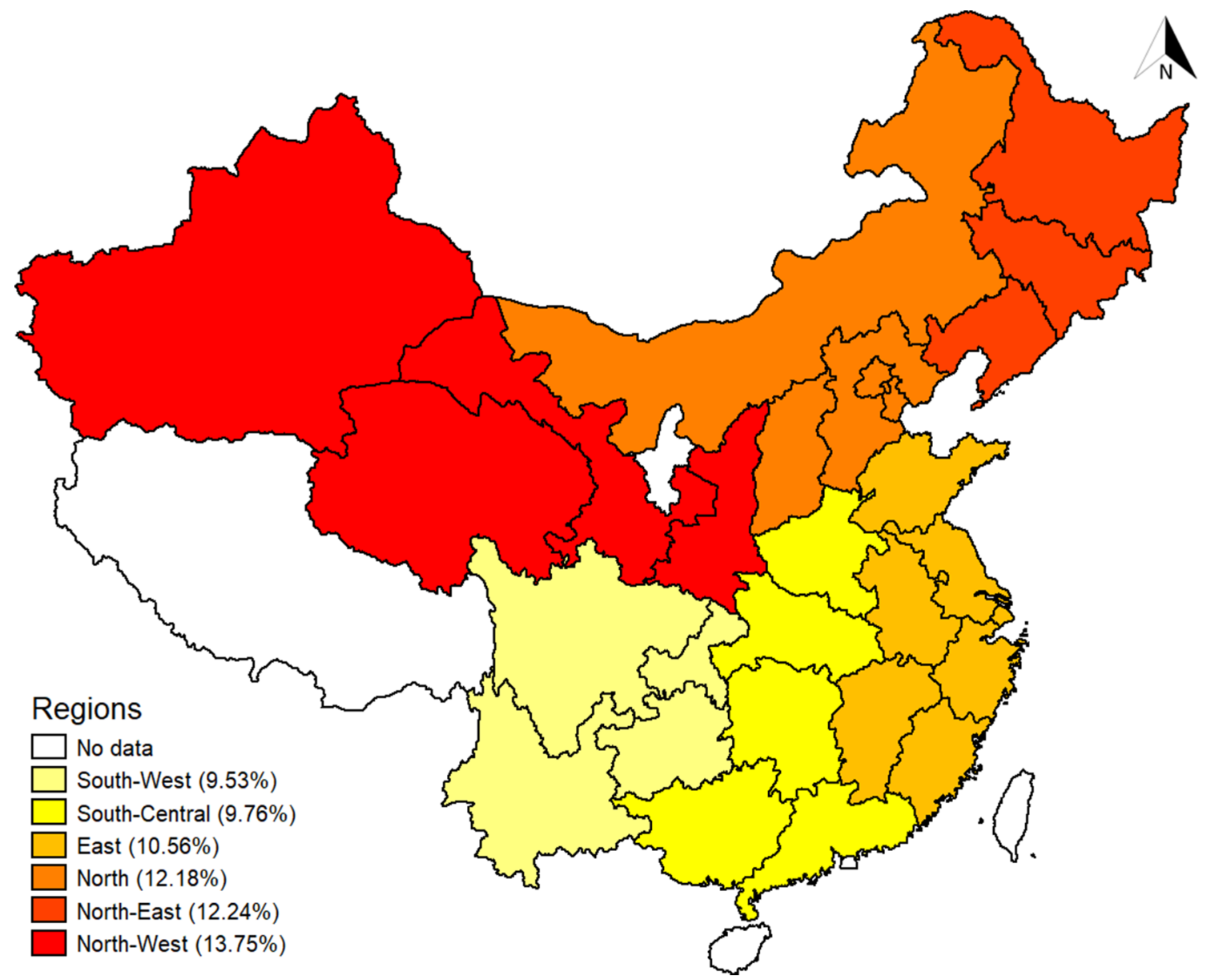

Figure 1 Prevalence in each region.

diagnosis than those in rural areas. We believe that there was inevitable selection bias, as residents living in urban areas may seek medical care more often than those in rural areas. Some individuals lack the recognition of BPH and hospital visitation is often delayed.

We demonstrated an apparent geographical variation in the prevalence of LUTS/BPH in China. Residents in the South-West regions had a much lower prevalence of LUTS/BPH than those living in the North-West regions of China. Individuals residing in the north regions, including North, North-East and North-West, had a relatively higher prevalence of LUTS/BPH than those living in South-Central and South-West. Dietary differences may contribute to this discrepancy. Daily diet of individuals living in the north regions contain much milk, dairy products and red meat, while individuals living in the south regions consume more fruits and vegetables than those in the north, which have both been confirmed to increase the risk of LUTS/BPH. ${ }^{29}{ }^{30}$ Nevertheless, residents in the East region did not show a high prevalence of LUTS/BPH. It was thought that socioeconomic development in the East region was, in general, higher than that of the other regions, possibly contributing to the lower prevalence of LUTS/BPH. Furthermore, the genetic susceptibility of $\mathrm{BPH}$ was also a factor influencing the prevalence of LUTS/BPH in China. Several studies from China reported genetics associated with developing $\mathrm{BPH},{ }^{30}$ possibly one of the factors causing the varying prevalence of LUTS/BPH in China.

Our research has several strengths. First, our data were based on CHARLS, a national population survey. The interviewers were highly trained and questionnaires were developed after a long and rigorous course. The participants were chosen via strict multistage probability sampling procedures. Hence, data from CHARLS can represent the national condition. Second, the prevalence of LUTS/BPH in China is difficult to estimate because of the large population and of the diagnostic criteria. Using data from CHARLS, we avoided both questions.

There are some limitations as well. First, CHARLS did not collect weight and height, so we could not analyse the relationship of body mass index or obesity and the prevalence of LUTS/BPH. Second, testicular androgens are required in the prostate for the development of BPH. Third, as China is a large country, and we all adhere to the principles of diagnosis from the China Urology Association, the diagnosis of LUTS/BPH may be slightly different among regions. We cannot detect these differences, since the data from CHARLS provide an established diagnosis.

In this study, we looked at the prevalence of LUTS/ $\mathrm{BPH}$ via CHARLS data. We found that the LUTS/BPH 
is highly prevalent in older, urban-living men. The prevalence varied according to marital status, socioeconomic status and geographical regions.

\section{Author affiliations}

${ }^{1}$ Urology, Peking University People's Hospital, Beijing, China

${ }^{2}$ Epidemiology and Health Statistics, Capital Medical University, Beijing, China

${ }^{3}$ Chinses PLA center for Disease Control and Prevention, Beijing, China

${ }^{4}$ Urology, Beijing Friendship Hospital Affiliated to Capital Medical University, Beijing, China

Acknowledgements The authors are full of gratitude to the office of CHARLS for sharing the data. The data can be accessed at the official website.

Contributors WZ, XZ: designed the study and drafted the article. HL, FW: dealt with the statistics. HW, MZ: helped with data sorting, literature retrieval, language polishing and other chores. $\mathrm{KX}, \mathrm{HH}$ : constructive revision and grasp of the overall situation.

Funding Supported By Peking University People's Hospital Research And Development Funds, Project (RDB2013-10).

Map disclaimer The depiction ofboundaries on the map(s) in this article do not imply the expression of anyopinion whatsoever on the part of BMJ (or any member of its group) concerningthe legal status of any country, territory, jurisdiction or area or of itsauthorities. The map(s) are provided without any warranty of any kind, eitherexpress or implied.

Competing interests None declared.

Patient consent for publication Obtained.

Ethics approval The project team filed an ethics review application to the Ethical Review Committee (IRB) at Peking University in June 2008 and obtained approval.

Provenance and peer review Not commissioned; externally peer reviewed.

Data sharing statement CHARLS is based on the Health and Retirement Study (HRS) and related ageing surveys such as the English Longitudinal Study of Ageing (ELSA) and the Survey of Health, Ageing and Retirement in Europe (SHARE). All of the data were obtained from CHARLS database (http://charls.pku.edu.cn/zh-CN) with open access.

Open access This is an open access article distributed in accordance with the Creative Commons Attribution Non Commercial (CC BY-NC 4.0) license, which permits others to distribute, remix, adapt, build upon this work non-commercially, and license their derivative works on different terms, provided the original work is properly cited, appropriate credit is given, any changes made indicated, and the use is non-commercial. See: http://creativecommons.org/licenses/by-nc/4.0/.

\section{REFERENCES}

1. Roehrborn CG. Pathology of benign prostatic hyperplasia. Int $J$ Impot Res 2008;20 Suppl 3:S11-S18.

2. Berry SJ, Coffey DS, Walsh PC, et al. The development of human benign prostatic hyperplasia with age. J Urol 1984;132:474-9.

3. Gu FL, Xia TL, Kong XT. Preliminary study of the frequency of benign prostatic hyperplasia and prostatic cancer in China. Urology 1994:44:688-91.

4. Lepor H. Pathophysiology of benign prostatic hyperplasia in the aging male population. Rev Urol 2005;7 Suppl 44:S3-S12.

5. Abrams P, Cardozo L, Fall M, et al. The standardisation of terminology of lower urinary tract function: report from the Standardisation Sub-committee of the International Continence Society. Neurourol Urodyn 2002;21:167-78.

6. Chapple CR, Roehrborn CG. A shifted paradigm for the further understanding, evaluation, and treatment of lower urinary tract symptoms in men: focus on the bladder. Eur Urol 2006;49:651-9.
7. Calais Da Silva F, Marquis P, Deschaseaux P, et al. Relative importance of sexuality and quality of life in patients with prostatic symptoms. Results of an international study. Eur Urol 1997:31:272-80.

8. Irwin DE, Milsom I, Hunskaar S, et al. Population-based survey of urinary incontinence, overactive bladder, and other lower urinary tract symptoms in five countries: results of the EPIC study. Eur Urol 2006;50:1306-15.

9. Maserejian NN, Chen S, Chiu GR, et al. Incidence of lower urinary tract symptoms in a population-based study of men and women. Urology 2013;82:560-4.

10. Dunphy C, Laor L, Te A, et al. Relationship between depression and lower urinary tract symptoms secondary to benign prostatic hyperplasia. Rev Urol 2015;17:51-7.

11. Wang W, Guo Y, Zhang D, et al. The prevalence of benign prostatic hyperplasia in mainland China: evidence from epidemiological surveys. Sci Rep 2015;5:13546.

12. Tabulation of the sixth national population census of the people's republic of china. http://www.stats.gov.cn/tjsj/pcsj/rkpc/6rp/indexch. htm.

13. Zhao Y, Hu Y, Smith JP, et al. Cohort profile: the China Health and Retirement Longitudinal Study (CHARLS). Int J Epidemiol 2014:43:61-8.

14. Research CCFE. China health and retirement longitudinal study: 2011-2012 national baseline blood data users' guide, 2014.

15. Chughtai B, Forde JC, Thomas DD, et al. Benign prostatic hyperplasia. Nat Rev Dis Primers 2016;2:16031.

16. Vuichoud C, Loughlin KR. Benign prostatic hyperplasia: epidemiology, economics and evaluation. Can J Urol 2015;22 Suppl $1: 1-6$

17. Egan KB. The epidemiology of benign prostatic hyperplasia associated with lower urinary tract symptoms: Prevalence and incident rates. Urol Clin North Am 2016;43:289-97.

18. Gandaglia G, Briganti A, Gontero P, et al. The role of chronic prostatic inflammation in the pathogenesis and progression of benign prostatic hyperplasia (BPH). BJU Int 2013;112:432-41.

19. Morote J, Lopez M, Encabo G, et al. Effect of inflammation and benign prostatic enlargement on total and percent free serum prostatic specific antigen. Eur Urol 2000;37:537-40.

20. Loeb S, Kettermann A, Carter HB, et al. Prostate volume changes over time: results from the baltimore longitudinal study of aging. $J$ Urol 2009:182:1458-62.

21. Roehrborn CG, Barkin J, Siami P, et al. Clinical outcomes after combined therapy with dutasteride plus tamsulosin or either monotherapy in men with benign prostatic hyperplasia (BPH) by baseline characteristics: 4-year results from the randomized, doubleblind Combination of Avodart and Tamsulosin (CombAT) trial. BJU Int 2011:107:946-54.

22. Platz EA, Joshu CE, Mondul AM, et al. Incidence and progression of lower urinary tract symptoms in a large prospective cohort of United States men. J Urol 2012;188:496-501.

23. Asiedu $B$, Anang $Y$, Nyarko $A$, et al. The role of sex steroid hormones in benign prostatic hyperplasia. Aging Male 2017;20:17-22.

24. Hutchison A, Farmer R, Chapple C, et al. Characteristics of patients presenting with LUTS/BPH in six European countries. Eur Urol 2006;50:555-62.

25. Glynn RJ, Campion EW, Bouchard GR, et al. The development of benign prostatic hyperplasia among volunteers in the Normative Aging Study. Am J Epidemiol 1985;121:78-90.

26. Araki $\mathrm{H}$, Watanabe $\mathrm{H}$, Mishina T, et al. High-risk group for benign prostatic hypertrophy. Prostate 1983;4:253-64.

27. Jo JK, Kim KS, Nam JW, et al. Sociodemographic factors related to lower urinary tract symptoms in men: A korean community health survey. Int Neurourol J 2017;21:143-51.

28. Fowke JH, Munro H, Signorello LB, et al. Association between socioeconomic status (SES) and lower urinary tract symptom (LUTS) severity among black and white men. J Gen Intern Med 2011;26:1305-10.

29. Lagiou P, Wuu J, Trichopoulou A, et al. Diet and benign prostatic hyperplasia: a study in Greece. Urology 1999;54:284-90.

30. Qi J, Tian L, Chen Z, et al. Genetic variants in $2 q 31$ and $5 p 15$ are associated with aggressive benign prostatic hyperplasia in a Chinese population. Prostate 2013;73:1182-90. 